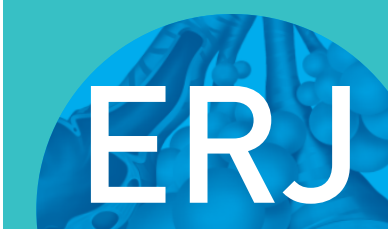

open research
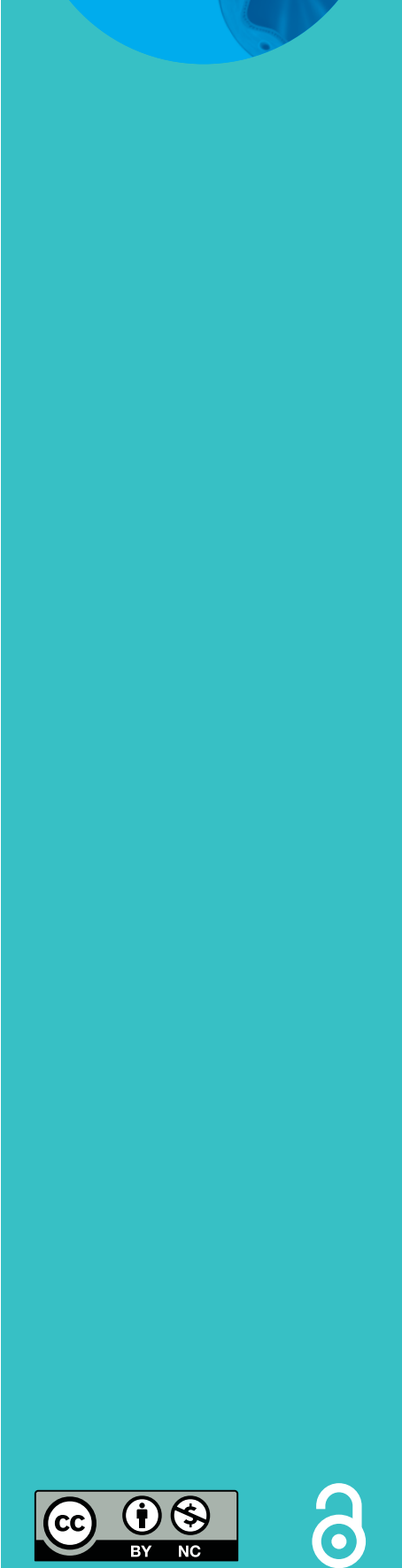

\section{The effect of adenotonsillectomy for childhood sleep apnoea on cardiorespiratory control}

\author{
Mathias Baumert ${ }^{1}$, Yvonne Pamula ${ }^{2}$, James Martin², Declan Kennedy ${ }^{2,3}$, \\ Anand Ganesan ${ }^{4}$, Muammar Kabir ${ }^{5}$, Mark Kohler ${ }^{3,6}$ and Sarah A. Immanuel ${ }^{1}$
}

Affiliations: 'School of Electrical and Electronic Engineering, University of Adelaide, Adelaide, Australia. ${ }^{2}$ Dept of Respiratory and Sleep Medicine, Women's and Children's Hospital, Adelaide, Australia. ${ }^{3}$ Childrens Research Centre, School of Paediatrics and Reproductive Health, University of Adelaide, Adelaide, Australia. ${ }^{4}$ Centre for Heart Rhythm Disorders, University of Adelaide and Royal Adelaide Hospital, Adelaide, Australia. ${ }^{5}$ Oregon Health \& Science University, Portland, OR, USA. ${ }^{6}$ School of Psychology, Social Work and Social Policy, University of South Australia, Adelaide, Australia.

Correspondence: Mathias Baumert, School of Electrical and Electronic Engineering, University of Adelaide, North Terrace, SA 5005, Australia. E-mail: mathias.baumertdadelaide.edu.au

ABSTRACT The efficacy of adenotonsillectomy for relieving obstructive sleep apnoea symptoms in children has been firmly established, but its precise effects on cardiorespiratory control are poorly understood.

In 375 children enrolled in the Childhood Adenotonsillectomy Trial, randomised to undergo either adenotonsillectomy $(n=194)$ or a strategy of watching waiting $(n=181)$, respiratory rate, respiratory sinus arrhythmia and heart rate were analysed during quiet, non-apnoeic and non-hypopnoeic breathing throughout sleep at baseline and at 7 months using overnight polysomnography.

Children who underwent early adenotonsillectomy demonstrated an increase in respiratory rate postsurgery while the watchful waiting group showed no change. Heart rate and respiratory sinus arrhythmia were comparable between both arms. On assessing cardiorespiratory variables with regard to normalisation of clinical polysomnography findings during follow-up, heart rate was reduced in children who had resolution of obstructive sleep apnoea syndrome, while no differences in their respiratory rate or respiratory sinus arrhythmia were observed.

Adenotonsillectomy for obstructive sleep apnoea increases baseline respiratory rate during sleep. Normalisation of apnoea-hypopnoea index, spontaneously or via surgery, lowers heart rate. Considering the small average effect size, the clinical significance is uncertain.

@ERSpublications

Adenotonsillectomy for childhood obstructive sleep apnoea increases respiratory rate during sleep http://ow.ly/Dptd300w9Pp

This article has supplementary material available from openres.ersjournals.com

Received: Jan 122016 | Accepted after revision: April 232016

Clinical trials: This study is registered at clinicaltrials.gov with identifier number NCT00560859.

Support statement: M. Baumert holds a fellowship from the Australian Research Council and this project was partly supported by grant ARC DP 110102049. The CHAT study was supported by grants (HL083075, HL083129, UL1-RR-024134 and UL1 RR024989) from the National Institutes of Health. Funding information for this article has been deposited with Open Funder Registry.

Conflict of Interest: None declared.

Copyright $\odot$ ERS 2016. This article is open access and distributed under the terms of the Creative Commons Attribution Non-Commercial Licence 4.0. 


\section{Introduction}

Upper airway obstruction is relatively common during childhood with a reported prevalence between $3 \%$ and $15 \%$ [1]. Its severity ranges from primary snoring to obstructive sleep apnoea syndrome (OSAS), with the majority of children showing symptoms at the milder end. Mounting evidence suggests that OSAS during childhood affects the cardiovascular system and may develop into cardiovascular disease later in life [2]. The autonomic nervous system is thought to mediate these changes. To this end, heart rate and respiratory sinus arrhythmia (RSA), i.e. the respiratory cycle-related modulation of the heart rate, can be utilised to probe sympathetic tone and vagal outflow to the heart $[3,4]$. Studies have suggested that the OSAS adversely affects autonomic nervous system activity in children [5-10].

As OSAS is often found in children with enlarged tonsils and adenoids, adenotonsillectomy is commonly the first-line treatment. The efficacy of adenotonsillectomy in treating the range of adverse health outcomes reported in children with OSAS, particularly for milder OSAS, has remained largely untested with additional concerns around post-surgical complications and the healthcare costs of performing large numbers of adenotonsillectomy [11-13]. Furthermore, evidence for the effects of adenotonsillectomy on cardiac autonomic control is inconclusive [14-17].

The aim of this study was to investigate the effects of adenotonsillectomy for treatment of OSAS on respiration and cardiac autonomic control by measuring respiratory rate, heart rate and RSA in school-age children, utilising data from the Childhood Adenotonsillectomy Trial (CHAT). The CHAT study is a landmark multicentre, controlled trial evaluating health and behavioural outcomes in children with OSAS randomised into early adenotonsillectomy (eAT) or watchful waiting. The study design and initial findings have been published elsewhere $[18,19]$.

\section{Methods}

Study sample

Detailed data of the CHAT protocol have been published previously [19]. All data are publicly available from https://sleepdata.org/datasets/chat In brief, children between 5 and 9.9 years of age with polysomnography (PSG) confirmed OSAS (apnoea-hypopnea index (AHI) $\geqslant 2$ events per hour or an obstructive apnoea index $(\mathrm{OAI}) \geqslant 1$ event per hour), a history of snoring and considered to be surgical candidates for adenotonsillectomy were recruited from paediatric sleep centres/sleep laboratories, paediatric otolaryngology clinics, general paediatric clinics and the general community from six clinical centres. Exclusion criteria included comorbidities, medications for psychiatric or behavioural disorders, recurrent tonsillitis, extreme obesity (body mass index (BMI) $>2.99$ for age group and sex z-score) and severe OSAS (AHI $\geqslant 30$ events. ${ }^{-1}$, OAI $\geqslant 20$ or oxyhaemoglobin saturation $<90 \%$ for $>2 \%$ of total sleep time). The study was approved by the Institutional Review Board of each institution. Informed consent was obtained from caregivers, and approval from children aged $\geqslant 7$ years. The study was registered at clinicaltrials.gov (NCT00560859).

\section{CHAT interventions}

Children were randomly assigned to either early adenotonsillectomy (surgery within 4 weeks after randomisation) or a strategy of watchful waiting with supportive care (WWSC) with reassessment of all the study variables at approximately 7 months. Complete bilateral tonsillectomy and removal of obstructing adenoid tissue was performed using standard surgical techniques. All children/caregivers received information on sleep hygiene using standardised educational materials that identified the need for regular sleep routines, age-appropriate sleep duration, avoidance of caffeine prior to bedtime, etc., and were given information on healthy lifestyle (nutrition and exercise). Children were also provided with saline nasal spray to be used nightly. At the baseline visit, children with coexisting conditions that could exacerbate the OSAS (e.g. allergies and poorly controlled asthma) were referred for treatment as needed.

\section{Overnight PSG}

Each child underwent in-laboratory baseline and follow-up PSG by study-certified technicians, following American Academy of Sleep Medicine paediatric guidelines for both acquisition and scoring [20]. The PSGs were centrally scored by registered sleep technicians. Overnight PSG was repeated approximately 7 months after randomisation [19].

\section{Neurophysiological tests and surveys}

The following neurophysiological outcomes were measured as part of the CHAT study. 1) Behaviour: by the parent's rating on the Conners' Parent Rating Scale-Revised: Long version Global Index (CGI ) t-score, a two-factor score comprising the restless impulsive and emotional-lability factor sets, and by the Behavior Rating Inventory of Executive Function (BRIEF) Global Executive Composite (GEC) t-score, comprising summary measures of behavioural regulation and metacognition. Teacher ratings from parallel instruments 
(the CGI short version and BRIEF Teacher Report Form) were also evaluated. 2) Symptoms of OSAS: by the total score of the Paediatric Sleep Questionnaire Sleep-Related Breathing Disorder Scale. 3) Sleepiness: the Epworth Sleepiness Scale modified for children. 4) Global quality of life: by the parental total score from the Paediatric Quality of Life Inventory, and disease-specific quality of life, assessed by the total score of the OSA-18, a composite of OSAS-related symptoms and quality of life. 5) The Differential Ability Scale II, a measure of generalised intellectual functioning.

\section{Cardiorespiratory analysis}

Cardiorespiratory analyses were conducted on all $30 \mathrm{~s}$ epochs of sleep data that were free from manually scored events (including respiratory events and periodic limb movements) and artefacts. RSA analysis was performed on ECG and thoracic respiratory inductance belt signals, using the phase-averaging technique proposed by GILAD et al. [21]. In brief, this method quantifies the magnitude of the R-R interval discursion in the ECG from its mean value with respect to the respiratory cycle. RSA was defined as the difference between the maximum and minimum values of the R-R curve averaged over multiple respiratory cycles. In addition, we measured the average respiratory rate from the thoracic respiratory inductance band and heart rate from the ECG. For details see the supplementary data.

\section{Statistical analysis}

Anthropometric data were compared using t-tests and Chi-squared tests as appropriate. Cardiorespiratory measures were analysed separately for stage 2 (N2) and slow wave sleep (N3) in non-rapid eye movement (NREM) sleep as well as REM sleep. A three-way ANCOVA was carried out for each variable under investigation with sleep stage as the repeated measure and study (baseline versus follow-up), as well as study arm (eAT versus WWSC). Anthropometric variables that were likely to confound statistical analysis (baseline BMI $z$-score, change in BMI $\mathrm{z}$-score, age, sex and race) were included in the model as covariates. Subsequently, a three-way ANCOVA was performed to test for the effect of AHI normalisation during follow-up (spontaneous or as a result of surgery; defined as AHI $\leqslant 2$ events $\cdot \mathrm{h}^{-1}$ and OAI $\leqslant 1$ events $\cdot \mathrm{h}^{-1}$ ) along with sleep stage and study factors. For post-hoc comparison, a one-way ANCOVA was performed on the follow-up data of measures that were significantly affected by AHI normalisation. Additional analysis included a one-way ANOVA to compare the effect of sleep stage on respiratory rate, heart rate and RSA, and Spearman correlation analysis to explore the relationship between percentage of total sleep time with partial pressure of carbon dioxide $>50 \mathrm{mmHg}$, percentage of sleep time with oxygen saturation $\leqslant 90 \%$ and respiratory rate, as well as associations between cardiorespiratory variables and outcomes of neurophysiological tests and surveys [22].

\section{Results}

\section{Subject demographics}

A total of 453 children from the original CHAT dataset were included in this study, comprising 194 children who underwent eAT and 181 children who joined the WWSC group and participated in both the baseline and follow-up sleep studies and had PSG that met the technical requirements (figure 1). Baseline anthropometric characteristics are summarised in table 1. Both groups had comparable demographic profiles. Overall, the mean age of the participants at baseline was 6.5 years and $48 \%$ were male. Approximately half (53\%) of the sample were African-American and $34 \%$ were obese. Around $5 \%$ of children were treated with montelukast and approximately $20 \%$ received glucocorticoids for rhinitis or asthma during baseline PSG. At follow-up, 82\% of subjects in the eAT group no longer had AHI-defined OSAS, i.e. AHI $\leqslant 2$ events $\cdot \mathrm{h}^{-1}$ and OAI $\leqslant 1$ events $\cdot \mathrm{h}^{-1}$, while in the WWSC group $42 \%$ of children had spontaneous normalisation of AHI scores. Approximately $8.2 \%$ of children in the eAT arm and $8.8 \%$ in the WWSC arm were on montelukast, and $24.1 \%$ and $27.1 \%$ (eAT and WWSC, respectively) were on glucocorticoids during follow-up PSG, representing a small, statistically nonsignificant increase compared with the baseline measurement.

\section{Effect of surgery on cardiorespiratory variables}

Our initial analysis evaluated the effect of surgery on cardiorespiratory variables by comparing the eAT group to the WWSC group. Respiratory rate in both groups was comparable at baseline but was significantly increased by an average of 1.3 cycles $\cdot \mathrm{min}^{-1}$ at 7 months in the children who underwent surgery but not in the WWSC group (table 2, figure 2).

To test if respiratory rate was associated with hypoventilation, correlation analysis with percentage of sleep with partial pressure of carbon dioxide $>50 \mathrm{mmHg}$ was performed and statistically significant inverse associations were observed in NREM sleep $(\mathrm{r}=-0.16, \mathrm{p}=0.002(\mathrm{~N} 2) ; \mathrm{r}=-0.15, \mathrm{p}=0.003(\mathrm{~N} 3) ; \mathrm{r}=-0.05$, $\mathrm{p}=0.2(\mathrm{REM}))$. Correlation analyses with percentage sleep time with oxygen saturation $\leqslant 90 \%$ were all nonsignificant $(\mathrm{r}=0.002(\mathrm{~N} 2), \mathrm{r}=0.02(\mathrm{~N} 3), \mathrm{r}=-0.001(\mathrm{REM}))$. 


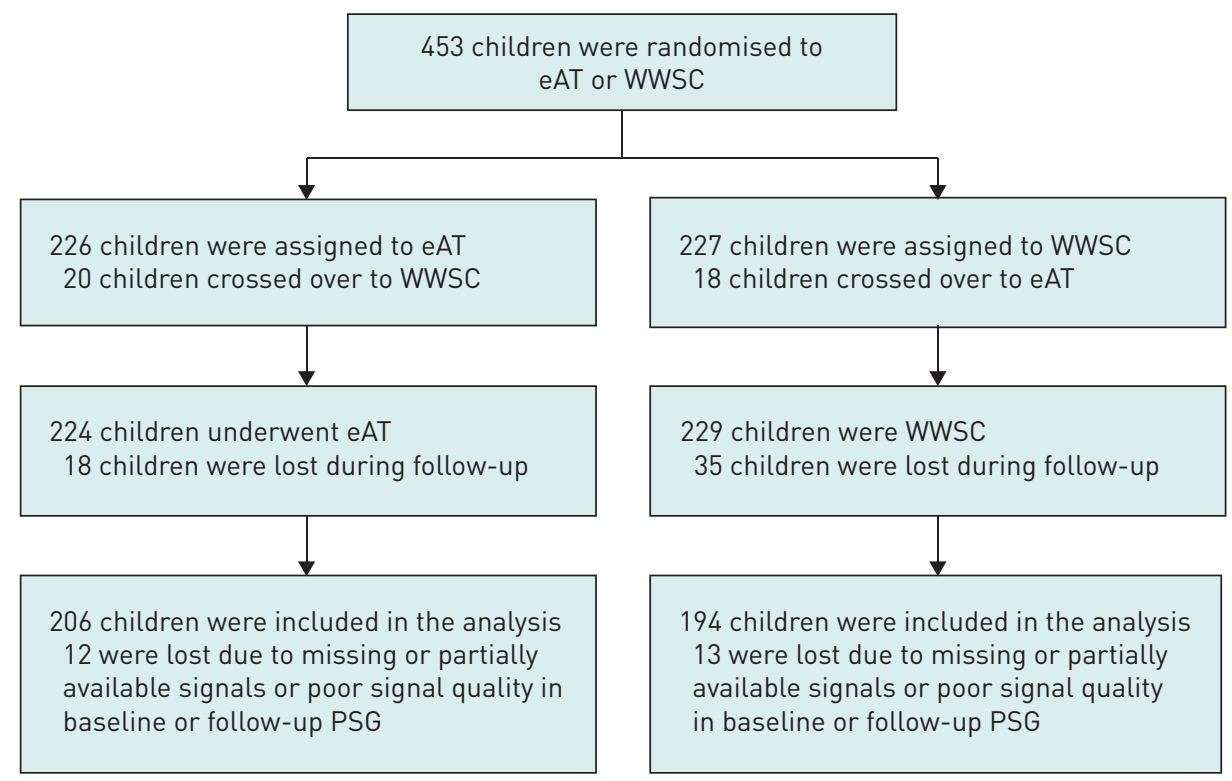

FIGURE 1 Summary of Childhood Adenotonsillectomy Trial study participants included in the cardiorespiratory analysis. eAT: early adenotonsillectomy; WWSC: watchful waiting with supportive care; PSG: polysomnography.

Heart rate was not significantly different within each group between the baseline and follow-up study and did not differ between groups at either time-point (table 2). RSA was comparable at baseline and not significantly different following surgery.

\section{Effect of AHI normalisation on cardiorespiratory variables}

As both study arms were heterogeneous with respect to AHI-defined OSAS normalisation, we stratified participants into those who had normal AHI scores at follow-up (either spontaneously or as a result of surgery, $\mathrm{n}=235)$ and those who still had AHI-defined OSAS $(\mathrm{n}=140)$ and investigated the effect of AHI normalisation per se on cardiorespiratory control (i.e. children were re-grouped with respect to subsequent normalisation of AHI). Anthropometric data were comparable except from the higher BMI z-score and a higher prevalence of overweight and obesity in children whose AHI did not normalise (table 1). The percentage of children who were

\section{TABLE 1 Baseline characteristics of subjects grouped according to study arm and}

apnoea-hypopnoea index (AHI) normalisation at 7 months

\begin{tabular}{|c|c|c|c|c|}
\hline \multirow[t]{2}{*}{ Characteristics } & \multicolumn{2}{|c|}{ Study arm } & \multicolumn{2}{|c|}{ AHI normalisation } \\
\hline & eAT & WWsc & Normalised & Not normalised \\
\hline Subjects & 194 & 181 & 235 & 140 \\
\hline Age years & $6.53 \pm 1.4$ & $6.58 \pm 1.3$ & $6.4 \pm 1.4$ & $6.7 \pm 1.3$ \\
\hline Male sex & $88(45 \%)$ & 94 (51\%) & $108(45 \%)$ & 72 (51\%) \\
\hline \multicolumn{5}{|l|}{ Race $^{\#}$} \\
\hline African-American & $100(51 \%)$ & $101(55 \%)$ & $108(46 \%)$ & $93(66 \%)$ \\
\hline Caucasian & 72 (37\%) & $65(35 \%)$ & $99(42 \%)$ & 37 (26\%) \\
\hline Other & $22(11 \%)$ & $16(9 \%)$ & $28(12 \%)$ & $10(7.9 \%)$ \\
\hline BMI z-score & $0.85 \pm 1.3$ & $0.92 \pm 1.2$ & $0.69 \pm 1.2$ & $1.2 \pm 1.2^{* * *}$ \\
\hline \multicolumn{5}{|l|}{ Weight class? } \\
\hline Overweight & 92 (47\%) & $88(48 \%)$ & $98(41 \%)$ & $81(57 \%)^{* *}$ \\
\hline Obese & $64(32 \%)$ & $64(35 \%)$ & $61(26 \%)$ & $66(47 \%)^{* * *}$ \\
\hline Montelukast & $9(4.6 \%)$ & $10(5.5 \%)$ & $8(3.4 \%)$ & $11(7.7 \%)$ \\
\hline Glucocorticoids & $40(20.6 \%)$ & $36(19.9 \%)$ & $48(20.4 \%)$ & $28(19 \%)$ \\
\hline
\end{tabular}




\section{TABLE 2 Comparison of cardiorespiratory measurements between time-points, intervention and sleep stage}

\begin{tabular}{|c|c|c|c|c|c|c|c|c|c|}
\hline & \multicolumn{2}{|c|}{ eAT } & \multicolumn{2}{|c|}{ wWsc } & \multicolumn{5}{|c|}{ p-value $\#$} \\
\hline & Baseline & Follow-up & Baseline & Follow-up & Time-point & Intervention & $\begin{array}{l}\text { Intervention } \\
\times \text { time-point }\end{array}$ & Sleep stage & $\begin{array}{l}\text { Intervention } \\
\text { x sleep stage }\end{array}$ \\
\hline N2 & $17.24 \pm 2.75$ & $18.60 \pm 2.46$ & $17.18 \pm 2.58$ & $17.52 \pm 2.68$ & $<0.001$ & 0.01 & 0.02 & $<0.001$ & $0.4^{+}$ \\
\hline \multicolumn{10}{|c|}{ 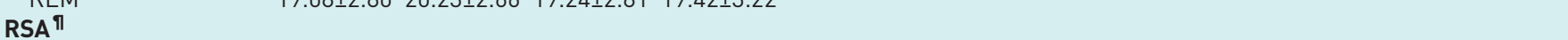 } \\
\hline N2 & $1.93 \pm 0.32$ & $1.86 \pm 0.2$ & $1.95 \pm 0.27$ & $1.95 \pm 0.27$ & $0.4^{+}$ & 0.06 & 0.05 & $<0.001$ & $0.5^{+}$ \\
\hline N3 & $1.90 \pm 0.35$ & $1.81 \pm 0.29$ & $1.90 \pm 0.30$ & $1.91 \pm 0.28$ & & & & & \\
\hline REM & $1.67 \pm 0.34$ & $1.59 \pm 0.32$ & $1.66 \pm 0.32$ & $1.67 \pm 0.31$ & & & & & \\
\hline \multicolumn{10}{|l|}{$\begin{array}{l}\text { Heart rate } \\
\text { beats per min }\end{array}$} \\
\hline \multicolumn{10}{|c|}{ 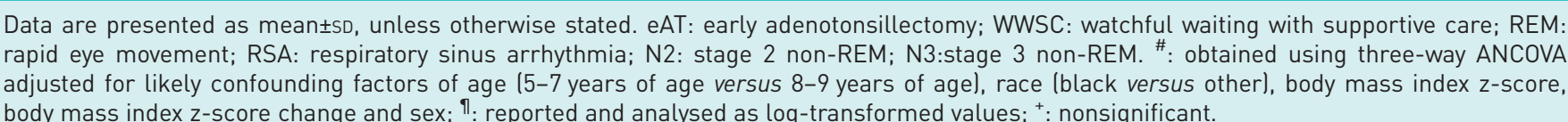 } \\
\hline
\end{tabular}

treated with montelukast or glucocorticoids at baseline were comparable between both groups. At follow-up, children whose AHI did not normalise were still more likely to be overweight/obese (65\% versus $48 \%$ ). The percentage of children who received montelukast (6.7\% versus $11.4 \%$ ) or glucocorticoids (24\% versus $27 \%$ ) remained comparable between children who normalised and those who did not.

Respiratory rate increased slightly in both groups of children from baseline to 7 months follow-up, but regardless of the assigned group respiratory rate and RSA were not significantly different between children who had normalisation of AHI and those who did not (table 3). During follow-up heart rate was significantly reduced on average by 2.46 beats. $\mathrm{min}^{-1}$ in children who had normal AHI scores compared to those whose scores remained elevated (figure 3a). To investigate if the effect of AHI normalisation on heart rate is different between children who underwent surgery and those who resolved spontaneously, a treatment arm-specific subgroup analysis of the follow-up data was performed, showing a significant normalisation effect within both groups (eAT: $\mathrm{p}=0.005$; WWSC: $\mathrm{p}=0.03$ ), but no significant between-group differences (figure $3 \mathrm{~b}$ ).

\section{Sleep stage effects on cardiorespiratory variables}

Our analysis showed a significant sleep stage effect on respiratory rate, heart rate and RSA (table 2). Post-hoc multiple comparisons showed that both respiratory rate and heart rate were significantly different in all three sleep stages $(\mathrm{p}<0.001)$, with REM sleep exhibiting the highest respiratory rate and heart rate. In

FIGURE 2 Average respiratory rate during sleep between the two study arms at baseline and 7-month follow-up. Data are presented as group means and standard error. eAT: early adenotonsillectomy; WWSC: watchful waiting with supportive care. ${ }^{* *}: p<0.01$.

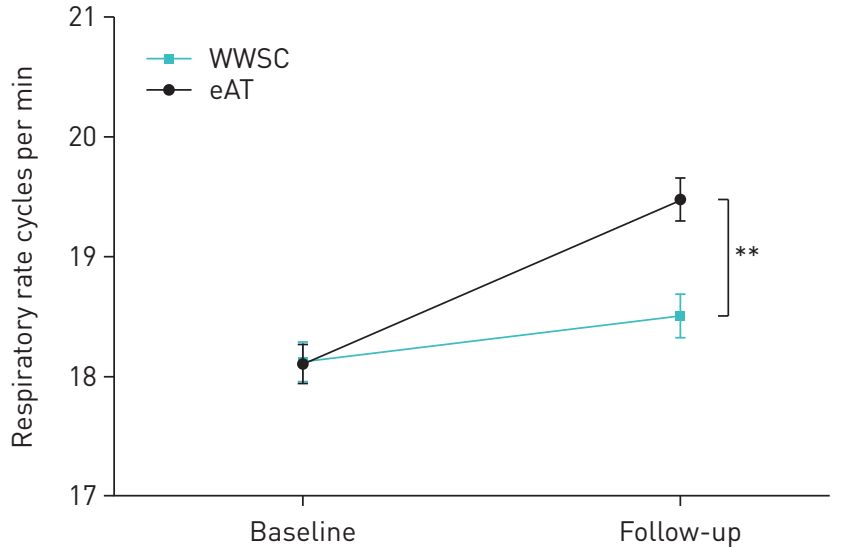


TABLE 3 Comparison of cardiorespiratory measurements between time-points, normalisation and sleep stage

\begin{tabular}{|c|c|c|c|c|c|c|c|c|}
\hline & \multicolumn{2}{|c|}{$\begin{array}{l}\text { OSA resolved at } \\
7 \text { months }\end{array}$} & \multicolumn{2}{|c|}{$\begin{array}{l}\text { OSA not resolved at } \\
7 \text { months }\end{array}$} & \multicolumn{4}{|c|}{ p-value } \\
\hline & Baseline & Follow-up & Baseline & Follow-up & Time-point & Normalisation & $\begin{array}{l}\text { Normalisation } \\
\times \text { time-point }\end{array}$ & Sleep stage \\
\hline Subjects & \multicolumn{2}{|c|}{235} & \multicolumn{2}{|c|}{140} & & & & \\
\hline \multicolumn{9}{|l|}{$\begin{array}{l}\text { Respiratory rate } \\
\text { cycles per } \\
\text { min }\end{array}$} \\
\hline N3 & $18.02 \pm 2.8$ & $18.86 \pm 2.6$ & $18.11 \pm 2.9$ & $19.04 \pm 3.1$ & & & & \\
\hline REM & $19.09 \pm 2.8$ & $19.9 \pm 2.9$ & $19.2 \pm 2.6$ & $19.5 \pm 3.0$ & & & & \\
\hline \multicolumn{9}{|l|}{ RSA $^{\text {T }}$} \\
\hline N2 & $1.96 \pm 0.29$ & $1.91 \pm 0.25$ & $1.91 \pm 0.32$ & $1.88 \pm 0.31$ & $0.4^{+}$ & $0.4^{+}$ & $0.7^{+}$ & $<0.001$ \\
\hline N3 & $1.92 \pm 0.31$ & $1.87 \pm 0.27$ & $1.87 \pm 0.34$ & $1.84 \pm 0.32$ & & & & \\
\hline REM & $1.67 \pm 0.32$ & $1.63 \pm 0.3$ & $1.65 \pm 0.33$ & $1.61 \pm 0.36$ & & & & \\
\hline REM & $84.8 \pm 9.1$ & $82.6 \pm 8.0$ & $85.5 \pm 8.7$ & $85.2 \pm 8.4$ & & & & \\
\hline \multicolumn{9}{|c|}{$\begin{array}{l}\text { Data are presented as mean } \pm \mathrm{SD} \text {, unless otherwise stated. OSA: obstructive sleep apnoea; REM: rapid eye movement; RSA: respiratory sinus } \\
\text { arrhythmia; N2: stage } 2 \text { non-REM; N3: stage } 3 \text { non-REM. \#: obtained using three-way ANCOVA adjusted for likely confounding factors of age } \\
\text { (5-7 years of age versus } 8-9 \text { years of age), race (black versus other), body mass index z-score, body mass index z-score change and sex; } \\
\overbrace{}^{\text {: }} \text { reported and analysed as log-transformed values; }{ }^{+}: \text {nonsignificant. }\end{array}$} \\
\hline
\end{tabular}

addition, RSA was significantly lower in REM sleep compared to N2 and N3 sleep. There was no significant sleep stage versus arm interaction effects for heart rate, respiratory rate and RSA measurements.

\section{Correlation between cardiorespiratory measures and neurophysiological tests and surveys}

Cardiorespiratory measures were not correlated with behaviour. Average heart rate was weakly inversely correlated with the Paediatric Quality of Life Total Scale Score from child self-reports (baseline: $r=-0.139$, $\mathrm{p}=0.006$; follow-up: $r=-0.114 ; \mathrm{p}=0.02$ ) and correlated with sleepiness measured by the Epworth Sleepiness Scale modified for children (baseline: $r=0.149, \mathrm{p}=0.004$; follow-up: $r=0.168 ; \mathrm{p}=0.001$ ), but not with the Paediatric Sleep Questionnaire Sleep-Related Breathing Disorder scale. Neither respiratory rate nor RSA were significantly correlated with OSAS symptoms, sleepiness or quality of life measures.

Generalised intellectual function measured by the Differential Ability Scale II consistently showed a weak inverse correlation with average respiratory rate (baseline: $r=-0.14, \mathrm{p}=0.006$; follow-up: $r=-0.128 ; \mathrm{p}=0.01$ ), but not with heart rate or RSA.
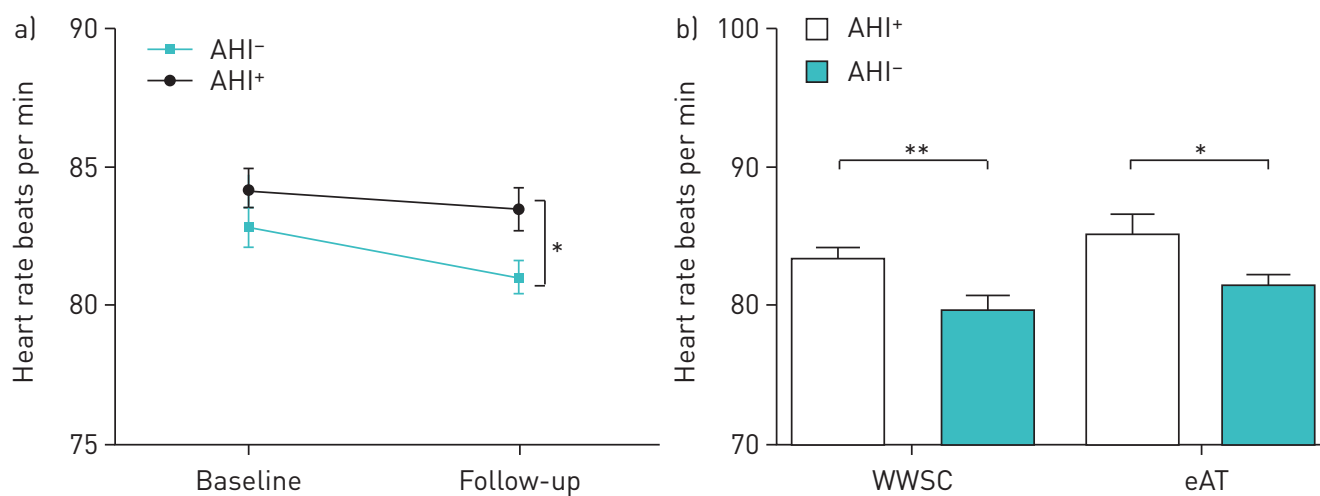

FIGURE 3 a) Mean heart rate during sleep for children who had normal apnoea-hypopnoea index (AHI) scores (either spontaneously or following adenotonsillectomyl versus those who still had polysomnography (PSG)-defined obstructive sleep apnoea syndrome. b) Subgroup analysis of heart rate during the follow-up PSG of children whose $\mathrm{AHI}$ score had normalised (AHI-) versus those whose $\mathrm{AHI}$ scores did not normalise $(\mathrm{AHI}+)$ within each arm. Data are presented as group means and standard error. eAT: early adenotonsillectomy; WWSC: watchful waiting with supportive care. ${ }^{*}: p<0.05 ;{ }^{* *}: p<0.01$. 


\section{Discussion}

Despite OSAS being common in childhood, critical questions remain unanswered with respect to sequelae, diagnosis and management. In particular the natural history of OSAS, the delineation of OSAS severity and treatment thresholds are poorly defined. The CHAT study was conducted to establish the efficacy for adenotonsillectomy in treating childhood OSA and to document the natural history (over a 7-month period) of untreated OSAS [19]. Although adenotonsillectomy was effective in treating a number of objective and subjective measures of OSAS in children, $21 \%$ of children who had eAT had residual OSAS at 7 months while a remarkable $46 \%$ of children in the WWSC group, who did not undergo surgery, had normal AHI scores after 7 months [18], raising questions about whom to treat and when. A set of secondary outcomes addressing cardio-metabolic health has been recently reported [15], where no significant intervention effect was observed for any of the measurements.

Our analysis provides evidence for the beneficial effect of treatment (eAT) on respiration in children with OSAS. At the 7-month follow-up, respiratory rate was significantly increased in all sleep stages in children who underwent eAT compared to the WWSC group. Mean heart rate during sleep remained unchanged across study time-points and between study arms, even after surgery in the eAT children. When children were stratified into those who normalised their AHI scores and those who did not, respiratory rate and RSA remained unchanged between groups at the follow-up, but heart rate was reduced in those children who no longer had abnormal AHI scores irrespective of whether this was achieved spontaneously or by adenotonsillectomy. These differential effects of treatment versus outcome are indicative of the heterogeneity in AHI normalisation across both study arms, suggesting that surgery resulted, on average, in slightly faster respiratory rate while the small heart rate reduction is primarily linked to normalisation of AHI scores.

In a previous study we found that respiratory rate was lower in children with predominantly mild OSAS compared to healthy children, with both inspiratory and expiratory time being prolonged during periods that were free of apnoea and hypopnea [23]. Following adenotonsillectomy, respiratory rate increased in these children and was no longer different from controls. Combined results suggest that surgery improves overall respiration in children with OSA independent of the frequency of frank obstructive events. The clinical significance of this rather small increase in respiratory rate is yet to be established. Although normalisation of the AHI score per se did not affect respiratory rate, it was inversely correlated with the percentage of total sleep time with carbon dioxide $>50 \mathrm{mmHg}$, suggesting that lower respiratory rate may result in hypercapnia during NREM sleep and point towards changes in the central control of respiratory drive. The increase in respiratory rate following adenotonsillectomy may also reflect the reduction in upper airway resistance [24]. Increased tissue mass as a result of adenotonsillar hypertrophy leads to a smaller airway diameter, which is an important determinant of upper airway resistance. As tidal volume was not measured, the net effect of adenotonsillectomy on minute ventilation remains unknown. An alternative clinical explanation might involve the improvement in asthma status following adenotonsillectomy. In a recent study, adenotonsillectomy was shown to reduce asthma exacerbations in children with OSAS [25]. At the baseline visit of the CHAT trial, children with coexisting conditions that could exacerbate the OSAS (e.g. allergies and poorly controlled asthma) were referred for treatment as needed.

The inverse association between respiratory rate and generalised intellectual functioning that, although weak in magnitude, was observed in both studies indicates a possible effect of baseline respiration during quite sleep on cortical processing. Pathophysiological pathways underpinning this relationship may involve hypoventilation, but warrant further investigation.

RSA is mediated by the vagus nerve and well-pronounced RSA is widely considered a marker of good cardiovascular fitness and health [26]. Our data suggest that adenotonsillectomy does not affect vagal heart rate control.

The significant but small decrease in resting heart rate that was observed, when comparing children who normalised in their clinical AHI scores versus children with persistent OSAS, suggest a possible beneficial effect of AHI normalisation on the heart. A significant association between a reduction in AHI indices and a reduction in average heart rate was previously shown in the CHAT data [15], which is in line with our findings. Resting heart rate is a marker of sympathetic tone [4] and, therefore, may suggest an organ-specific or systemic decrease in sympathetic outflow following the resolution of OSAS. Significant correlations between average heart rate and sleepiness, as well as child-reported reduction in quality of life, suggest a link between sympathetic activity and commonly observed daytime symptoms of OSAS. Previous studies have shown a significant reduction in heart rate in children with OSAS post adenotonsillectomy [16, 17], while a study comparing heart rate in children whose OSAS resolved versus those that did not resolve after a 4-year follow-up did not find significant between-group differences [27].

As has already been highlighted by the CHAT study investigators, the children in this study had only mild-to-moderate OSAS as defined by AHI and the follow-up time was relatively short. More severe OSAS 
is likely to augment autonomic nervous system disturbances. Although our results are based on a large randomised control trial with racially diverse groups, including standardised measurements and high follow-up rates, there are limitations with respect to their interpretation. Several of the recordings from the original study were omitted due to poor signal quality. In absence of calibrated inductance signals, which would provide a semi-quantitative measure of tidal volume, assessment of respiratory function was solely based on respiratory rate. Furthermore, children in the WWSC group who showed spontaneous resolution of OSAS at 7 months tended to be non-African-American, non-obese and had lower baseline AHI [28]. Thus by re-stratifying children into those with and without resolution of OSAS may have resulted in two groups with different clinical and demographic characteristics. Drugs affecting vagal tone (e.g. montelukast) may have affected our results, but the number of children receiving montelukast was small and not significantly different between baseline and follow-up PSG.

In conclusion, adenotonsillectomy for childhood OSAS increases baseline respiratory rate during sleep. Normalisation of AHI scores, spontaneously or via surgery, results in lower heart rate. As both changes are on average small, their clinical significance is uncertain; they may help maintain long-term cardiovascular health.

\section{Acknowledgements}

We would like to thank Michael Rueschman (Division of Sleep and Circadian Disorders, Brigham and Women's Hospital, Boston, MA, USA) for support with handling and interpreting the CHAT dataset. Mathias Baumert and Sarah A. Immanuel had full access to all of the data in the study and take responsibility for the integrity of the data and accuracy of the data analysis. Yvonne Pamula, Muammar Kabir, Declan Kennedy, James Martin, Mark Kohler and Anand Ganesan contributed substantially to the interpretation and writing of the manuscript.

\section{References}

1 Lumeng JC, Chervin RD. Epidemiology of pediatric obstructive sleep apnea. Proc Am Thorac Soc 2008; 5: $242-252$.

2 Nisbet LC, Yiallourou SR, Walter LM, et al. Blood pressure regulation, autonomic control and sleep disordered breathing in children. Sleep Med Rev 2014; 18: 179-189.

3 Eckberg DL. Human sinus arrhythmia as an index of vagal cardiac outflow. J Appl Physiol Respir Environ Exerc Physiol 1983; 54: 961-966.

4 Grassi G, Vailati S, Bertinieri G, et al. Heart rate as marker of sympathetic activity. J Hypertens 1998; 16 $1635-1639$.

5 Nisbet LC, Yiallourou SR, Nixon GM, et al. Nocturnal autonomic function in preschool children with sleep-disordered breathing. Sleep Med 2013; 14: 1310-1316.

6 Liao D, Li X, Rodriguez-Colon SM, et al. Sleep-disordered breathing and cardiac autonomic modulation in children. Sleep Med 2010; 11: 484-488.

7 Kwok KL, Ng DK, Chan $\mathrm{CH}$. Cardiovascular changes in children with snoring and obstructive sleep apnoea. Ann Acad Med Singapore 2008; 37: 715-721.

8 Walter LM, Yiallourou SR, Vlahandonis A, et al. Impaired blood pressure control in children with obstructive sleep apnea. Sleep Med 2013; 14: 858-866.

9 Baharav A, Kotagal S, Gibbons V, et al. Fluctuations in autonomic nervous activity during sleep displayed by power spectrum analysis of heart rate variability. Neurology 1995; 45: 1183-1187.

10 Aljadeff G, Gozal D, Schechtman VL, et al. Heart rate variability in children with obstructive sleep apnea. Sleep 1997; 20: 151-157.

11 Brietzke SE, Gallagher D. The effectiveness of tonsillectomy and adenoidectomy in the treatment of pediatric obstructive sleep apnea/hypopnea syndrome: a meta-analysis. Otolaryngol Head Neck Surg 2006; 134: 979-984.

12 Bhattacharjee R, Kheirandish-Gozal L, Spruyt K, et al. Adenotonsillectomy outcomes in treatment of obstructive sleep apnea in children: a multicenter retrospective study. Am J Respir Crit Care Med 2010; 182: 676-683.

13 Costa DJ, Mitchell R. Adenotonsillectomy for obstructive sleep apnea in obese children: a meta-analysis. Otolaryngol Head Neck Surg 2009; 140: 455-460.

14 Vlahandonis A, Walter LM, Horne RS. Does treatment of SDB in children improve cardiovascular outcome? Sleep Med Rev 2013; 17: 75-85.

15 Quante M, Wang R, Weng J, et al. The effect of adenotonsillectomy for childhood sleep apnea on cardiometabolic measures. Sleep 2015; 38: 1395-1403.

16 Muzumdar HV, Sin S, Nikova M, et al. Changes in heart rate variability after adenotonsillectomy in children with obstructive sleep apnea. Chest 2011; 139: 1050-1059.

17 Constantin E, McGregor CD, Cote V, et al. Pulse rate and pulse rate variability decrease after adenotonsillectomy for obstructive sleep apnea. Pediatr Pulmonol 2008; 43: 498-504.

18 Marcus CL, Moore RH, Rosen CL, et al. A randomized trial of adenotonsillectomy for childhood sleep apnea. N Engl J Med 2013; 368: 2366-2376.

19 Redline S, Amin R, Beebe D, et al. The Childhood Adenotonsillectomy Trial (CHAT): rationale, design, and challenges of a randomized controlled trial evaluating a standard surgical procedure in a pediatric population. Sleep 2011; 34: 1509 .

20 Berry RB, Budhiraja R, Gottlieb DJ, et al. Rules for scoring respiratory events in sleep: update of the 2007 AASM Manual for the Scoring of Sleep and Associated Events. Deliberations of the Sleep Apnea Definitions Task Force of the American Academy of Sleep Medicine. J Clin Sleep Med 2012; 8: 597-619.

21 Gilad O, Swenne CA, Davrath LR, et al. Phase-averaged characterization of respiratory sinus arrhythmia pattern. Am J Physiol Heart Circ Physiol 2005; 288: H504-H510.

22 Marcus CL, Moore RH, Rosen CL, et al. A randomized trial of adenotonsillectomy for childhood sleep apnea. N Engl J Med 2013; 368: 2366-2376. 
23 Immanuel SA, Pamula Y, Kohler M, et al. Respiratory timing and variability during sleep in children with sleep-disordered breathing. J Appl Physiol 2012; 113: 1635-1642.

24 Smith PL, Wise RA, Gold AR, et al. Upper airway pressure-flow relationships in obstructive sleep-apnea. J Appl Physiol 1988; 64: 789-795.

25 Bhattacharjee R, Choi BH, Gozal D, et al. Association of adenotonsillectomy with asthma outcomes in children: a longitudinal database analysis. PLoS Med 2014; 11: e1001753.

26 Demeersman RE. Heart-rate-variability and aerobic fitness. Am Heart J 1993; 125: 726-731.

27 Vlahandonis A, Yiallourou SR, Sands SA, et al. Long-term changes in blood pressure control in elementary school-aged children with sleep-disordered breathing. Sleep Med 2014; 15: 83-90.

28 Chervin RD, Ellenberg SS Hou, X, et al. Prognosis for spontaneous resolution of OSA in children. Chest 2015; 148: $1204-1213$. 